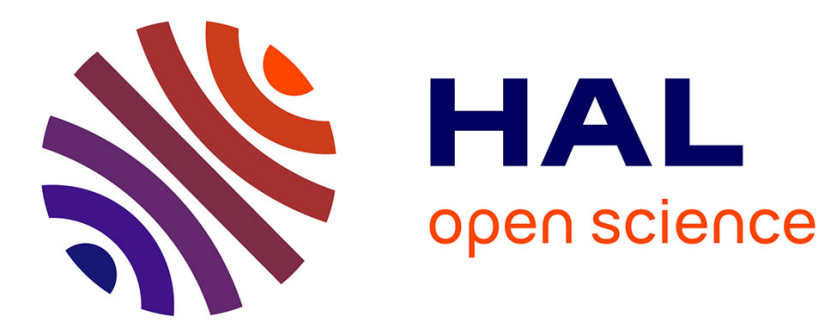

\title{
Produits chimiques, sylviculture et reboisement Pierre Arbonnier
}

\section{To cite this version:}

Pierre Arbonnier. Produits chimiques, sylviculture et reboisement. Revue forestière française, 1959, 8-9, pp.605-609. 10.4267/2042/27531 . hal-03382122

\section{HAL Id: hal-03382122 \\ https://hal.science/hal-03382122}

Submitted on 18 Oct 2021

HAL is a multi-disciplinary open access archive for the deposit and dissemination of scientific research documents, whether they are published or not. The documents may come from teaching and research institutions in France or abroad, or from public or private research centers.
L'archive ouverte pluridisciplinaire HAL, est destinée au dépôt et à la diffusion de documents scientifiques de niveau recherche, publiés ou non, émanant des établissements d'enseignement et de recherche français ou étrangers, des laboratoires publics ou privés. 


\section{PRODUITS CHIMIQUES SYLVICULTURE ET REBOISEMENT}

\section{L'utilisation des auxines pour l'élagage artificiel}

L'existence de branches basses ou l'apparition de gourmands sur le fût constituent toujours pour l'arbre un élément de dépréciation. Le déclassement de toute la grume ou d'une partie de la bille de pied est particulièrement préjudiciable au producteur lorsqu'il intéresse des essences pouvant fournir des bois de qualité exceptionnelle (tranchage ou déroulage) atteignant des prix très élevés.

Il n'est donc pas étonnant que le forestier se préoccupe de plus en plus d'assurer aux tiges d'avenir des peuplements en croissance un élagage soigné. Il dispose pour cela de deux moyens: favoriser l'élagage naturel, pratiquer l'élagage artificiel.

L'élagage naturel s'obtient en conduisant les peuplements à l'état serré. Cette méthode, apparemment la moins onéreuse, ne peut être pratiquée que dans certaines limites. Elle est souvent contrariée par la nécessité d'obtenir, au terme de la vie du peuplement, (les semenciers efficaces, donc pourvus de cimes suffisamment développées. En certains cas, soit qu'il s'agisse de conditions de milieu (altitude, hygrométrie, etc...) ou des caractères propres de l'espèce, de la race ou de l'individu, l'élagage naturel, même à l'état serré, se fait mal. Enfin, on ne peut l'adopter pour certaines essences plantées à larges espacements, notamment les peupliers.

L'élagage artificiel a de nombreux défenseurs et fait encore l'objet de nombreuses études. Pourtant, dès qu'on sectionne un rameau vert, on ouvre au flanc de l'arbre une blessure par laquelle les parasites pourront facilement pénétrer. L'idéal est bien de provoquer artificiellement la mort du rameau avant de le sectionner, laissant aux mécanismes vitaux de l'arbre le soin de préparer la décurtation par une cicatrisation interne analogue à celle qui se produit dans l'élagage naturel.

Tel est le but que l'on est tenté de rechercher en utilisant les auxines de synthèse et plus particulièrement le 2.4.5.T.

L'idée est en soi fort séduisante. On sait que les auxines n'nnt pas dans le végétal une faculté de diffusion illimitée. La diffusion est essentiellement verticale, souvent aussi radiale (I) mais très faible dans le sens tangentiel. Cette propriété est utilisée notamment pour la taille (artificielle) des haies. De plus, on peut penser 
que sur un végétal de grandes dimensions, l'application localisée de faibles quantités d'auxines n'aura d'effet qu'au voisinage immédiat des parties traitées.

Plusieurs forestiers allemands se sont ainsi attachés à vérifier ces hypothèses et à mettre au point une technique d'application pratique. Nous citerons pour mémoire les-essais infructueux de FrömMSDORFF mentionnés par MAYER-WEGELIN (3) et qui faisaient appel à une solution de chlorate de soude. L'idée d'utiliser les auxines revient, semble-t-i1, à Splettstösser (4), qui dès I95I, entreprit des essais sur le chêne à l'aicle de Tormon (*) avec le con cours de la firme Cena qui fabriquait ce produit. Les essais furent développés en I953 dans un peuplement de 50 ans où, sur un hectare, cent vingt tiges d'élite furent traitées. Les résultats étant apparemment satisfaisants, les traitements furent encore amplifiés en I954 (dix hectares traités) et poursuivis en I955 et I956 avec le Tormona 80 , en faisant varier la concentration du produit et l'âge des tiges traitées. Toutes ces expériences ont permis de connaitre, avec un recul suffisant, les effets des traitements.

En règle générale, après pulvérisation on observe un desséchement assez rapide du rameau traité qui perd ses feuilles en l'espace de quelques semaines. Deux ou trois ans plus tard, le ramean s'est détaché naturellement, ou peut être très facilement séparé du tronc. Quelques nouveaux goumands peuvent apparaitre, mais sans ancune vigueur et il est facile cle leur appliquer le même traitement. Quant à l'arbre, il ne parait pas affecté dans sa croissance. Sa cime reste normale.

Apparemment donc le phénomène est très proche de celui qu'on observe dans l'élagage naturel. Mais il était important de connaître quelles sont les modifications éventuelles qui peuvent se produire dans la composition dı bois au point d'insertion dı gourmand détruit (pourriture, coloration, etc...). En I956, des billons prélevés sur les arbres traités en I95.3 et I955 furent confiés pour examen au Prof. Mayer-Wegeris à l'Institut de Hann-Munden. Le diagnostic de ce spécialiste fut pleinement satisfaisant. L'examen permit en effet de confirmer la formation à la base du rameau d'une couche de protection cicatricielle. Cette couche avait atteint I à $2 \mathrm{~mm}$ un an après le traitement et davantage avec un recul plus important (I953). Par ailleurs, aucune altération du bois qui puisse être imputée au traitement chimique n'a été relevée. Le Prof. Mayer-Wegelin conclut donc à la pleine efficacité de la technique.

Fort de ces conclusions, Splettstösser définit comme suit les modalités d'emploi de l'élagage chimique:

(*) Splftrstösser n'indique pas la composition du Tormon. Mais les essais ont abouti à la formulation du Tormona 80 dont l'élément actif est un ester du 2.4.5.T (CELA - Ingelheim am Rhein). 
Age des peuplements. - L'âge minimum est d'environ 30 ans, ce qui correspond, selon l'auteur, à l'apparition de crevasses sur l'écorce. Il n'y a pas de linite d'âge supérieure, mais il n'apparait pas souhaitable d'élaguer des chênes dépassant un diamètre (au milieu) de $20 \mathrm{~cm}$, si l'on veut que la couche de bois sans défaut cle la future grume atteigne une épaisseur suffisante. Corrélativement à la notion d'âge minimum, il n'est pas indiqué d'élaguer des arbres dont la hauteur riatteint pas une douzaine de mètres, l'élagage (généralement sur les six premiers mètres) risquant alors d'amputer trop sérieusement la cime et d'affecter la croissance du sujet.

Nombre de tiges à l'hectare. - Il dépend évidemment de l'objectif économique recherché (et aussi, selon nous, de la station). SPLETTSTÖSSER préconise 200 tiges/hectare, bien que ce chiffre soit supérieur au nombre de tiges atteignant 1'âge de la révolution. I1 est presque certain en effet que le choix opéré dans un peuplement jeune ne demeurera pas définitif et il est bon de garder une petite marge de manœuvre aux exécutants ultérieurs des martelages.

Pćriode de traitement. - Comme chaque fois qu'il s'agit d'auxines, en pleine période de végétation, de juin à août, par temps chaud et en l'absence de vent.

Matériel. - Splettstösser a utilisé des pulvérisateurs à dos de I 5 litres avec canne-rallonge de 2 à $3 \mathrm{~m}$ permettant de traiter jusqu'à une hauteur de 6 mètres (*). Equipage de deux hommes, l'un manipulant l'appareil et l'autre maniant la lance. Il importe de bien arroser tout le feuillage du gourmand à détruire.

Consommation. - Un litre par arbre d'une solution à $0,2 \%$ de Tormona $80\left(^{*}\right)$, soit 200 'litres d'eau et moins d'un demi-litre de Tormona 80 à l'hectare. La main-d'œuvre est évaluée à vingt heures d'ouvrier.

Sur la base de ces données, compte tenu du prix actuel de l'heure d'ouvrier forestier et en admettant pour le Tormona 80 un prix comparable à celui du $\mathrm{P}$. 80 - dont la composition et la con-

(*) Cette hauteur pourra paraitre chez nous insuffisante en certain cas. Elle est pratiquement liée à la pression disponible à la sortie de l'appareil à dos. On peut cependant envisager de faire appel, pour les pulvérisations, à un matériel plus important. I1 existe à l'heure actuelle des groupes tractés ou portés suffisamment.maniables et disposant de possibilités de réglage de la pression par by-pass. De tels appareils sont parfaitement utilisables pour obtenir des hauteurs d'élagage supérieures à 6 mètres.

(*) Le Tormona 80 contient $80 \%$ d'ester amylique de l'acide $2.4 .5-\mathrm{T}$, soit $757 \mathrm{~g} / 1$ d'équivalent acide. La concentration indiquée correspond sensiblement à 2 pour mille de $\mathrm{P} .80$ ( $7 \mathrm{lo} \mathrm{g} / 1$ éq. ac.) et à 3 pour mille de Sylvoxone 60 (480 g/1 éq. 'ac.). 
centration diffèrent peu - on peut estimer à $5000 \mathrm{~F}$ l'hectare environ le prix d'un élagage chimique.

$\mathrm{Au}$ bout de quatre ans, un second traitement - moins coûteux - peut être nécessaire pour éliminer les petits gourmands réapparus sur les tiges traitées. On peut en profiter pour détacher les rameaux morts qui ne se seraient pas naturellement séparés du tronc après le traitement initial. La coupe des rameaux secs peut du reste intervenir plus tôt, puisqu'il a été prouvé que les tissus cicatriciels sont déjà formés au bout d'un an.

Les résultats obtenus sur le chêne par Splettstösser ont naturellement incité certains chercheurs à expérimenter l'élagage artificiel sur d'autres essences.

Comme il fallait s'y attendre, les résultats ne sont pas très encourageants sur les résineux. On sait que ceux-ci manifestent à l'égard des auxines et notamment du 2.4 .5 -T une résistance nettement supérieure à celle de la plupart des feuillus. Cette propriété est du reste exploitée aujourd'hui dans les dégagements sélectifs des plantations résineuses, technique dont nous entretiendrons prochainement le lecteur.

Mais parmi les espèces particulièrement sensibles aux auxines, il en est pour lesquelles la pratique de l'élagage est toujours nécessaire: les peupliers. L'expérimentation de l'élagage chimique de cette essence a été entreprise par Walter Liese (2). Précisons tout de suite qu'elle ne paraît pas encore avoir clonné des résultats définitifs.

Les premières tentatives ont porté, en août I956, sur la destruction des branches latérales de sujets âgés de 2 à 7 ans et sur rejets rle souches de $\mathrm{I}$ et 2 ans, avec le Tormona 80 à diverses concentrations.

Les premières observations ont permis de noter une nécrose rapide des feuilles qui presque toutes étaient tombées fin septembre. Seuls quelques rameaux traités à 0, I $5 \%$ conservèrent une partie de leurs feuilles et reverdirent à nouveau au printemps suivant. Cette concentration apparaît donc comme la limite courte des dosages efficaces.

Les observations du printemps suivant sur les rameaux ou rejets traités aux concentrations de I et $2 \%$ - qui nous paraissent excessives pour des pulvérisations - laissèrent constater le desséchement total cles parties traitées, sans que les rameaux respectés par la pulvérisation paraissent souffrir. La séparation entre tissus morts et tissus vivants se caractérisait alors par une solution de continuité nettement !perceptible au changement de couleur et de texture le l'écorce. Ce phénomène est à peu près constant sur toutes les espèces ligneuses touchées par les auxines. De plus, les rejets sont 
souvent atteints au point d'insertion sur le collet de tumeurs analogues à de petits chancres et se détachent de la souche sans effort.

Comme dans les essais de Splettstösser, des prélèvements de billons eurent lieu en 1957 sur les tiges traitées et l'on a pu là encore constater l'absence de toute altération susceptible de déprécier le bois.

L'attention des observateurs fut cependant attirée par la décomposition rapide des rameaux traités sur lesquels ne tardèrent pas à apparaitre des fructifications de champignons parasites du peuplier: Cytospora Chrysosperma, C. nivea et Dotichiza populea, indiquant une contamination précoce remontant sans doute à l'automne précédent... Il y a là un grand danger, car ces champignons peuvent, en conditions favorables, ne plus se borner à attaquer des tissus affaiblis et, d'endémiques, devenir épidémiques.

Les expériences préliminaires de Liese ont donc montré qu'il était parfaitement possible d'élaguer chimiquement le peuplier, mais laissent planer un doute sur l'opportunité d'une telle pratique qui risque de créer, au sein des plantations, des foyers de développement de parasites pouvant devenir virulents. Seuls, un recul suffisant et de nouvelles expériences (notamment avec des solutions plus diluées, des concentrations de l'ordre de 0,2 à $0,3 \%$ nous paraissant plus indiquées) permettront de jauger le risque que le développement des parasites fait courir à la peupleraie.

Enfin, il est également possible d'étendre à d'autres essences feuillues la technique inaugurée par SPLetTSTössER. L'avantage essentiel de celle-ci nous parait résider dans la stimulation artificielle de la formation de tissus cicatriciels permettant la décurtation ultérieure sans création de plaies.

Nous ne pensons pas que des expériences analogues à celles qui ont été relatées ci-dessus aient eu lieu jusqu'à présent en France. L'ampleur de ses tâches n'a pas encore pernis à la $I^{\text {re }}$ Section de la Station de Recherches de s'attaquer à ce problème. En faisant connaitre les essais - et les résultats - de Splettstösser et de I.IESE nous espérons susciter des expérimentateurs bénévoles à qui notre aide est acquise d'avance.

P. Arbonnier,

I $^{\text {re }}$ Section de la Station de Recherches.

\section{BIBLIOGRAPHIE}

I. Cherng, Jian Shine, R.L. Hossfeld, L. W. Rees. - Absorption and translocation of 2.4.5-trichlorophenoxyacetic acid derivatives in Quaking Aspen. Forest Science, vol. 4, n ${ }^{\circ}$ I, déc. I958, p. 319-323.

2. Liese Walter. - Orientierende Untersuchungen zur chemischen Astung von Pappeln. Der Forst-und Holzwirt, $\mathrm{n}^{\circ} \mathrm{I} 7 \mathrm{I}^{\mathrm{er}}$ septembre 1957, 2 p.

3. Mayer-Wegelin H. - Die neuere Entwicklung der Technik des Aufästens. Allgemeine Forstzeitschrift, $\mathrm{n}^{\circ}$ 22, 30 mai 1959, p. 397-400.

4. SPlettstösSer. - Asten von Eichen mit Wuchsstoffen. Der Forst- und Holzwirt, $\mathrm{n}^{\circ} 8$, $\mathrm{i} 6$ avril $\mathrm{1957,}, \mathrm{p}$. 